\title{
Uso del suelo y estado de conservación de la vegetación leñosa del monte en el noreste patagónico
}

\author{
Juan M. Zeberio ${ }^{\prime \bowtie ;}$; Silvia S. Torres Robles' \& Graciela M. Calabrese² \\ ${ }^{1}$ Universidad Nacional de Río Negro, Sede Atlántica. Río Negro, Argentina. ${ }^{2}$ Universidad Nacional de Río Negro, Sede \\ Andina. Río Negro, Argentina.
}

\begin{abstract}
Resumen. A menudo, la productividad de los sistemas naturales es manejada para obtener bienes y servicios ambientales. El principal uso de los ecosistemas semiáridos del NE de la Patagonia está orientado hacia la ganadería extensiva. Esta actividad se desarrolla en áreas cubiertas por vegetación nativa (estepas arbustivograminosas con bosquetes más o menos aislados de especies arbóreas). El objetivo de este trabajo fue relacionar aspectos estructurales, composicionales y del uso antrópico de las comunidades del monte del NE de la Patagonia. Se caracterizó a las comunidades de plantas vasculares mediante parámetros ecológicos como el índice de Shannon-Wiener $\left(\mathrm{H}^{\prime}\right)$, el índice de valor de importancia (IVI) y la cobertura por estratos. Los sitios en los que los estratos arbustivos alto y bajo proyectan coberturas similares y en los que domina la cobertura del estrato arbustivo alto presentaron los valores más altos del índice de Shannon, una cobertura elevada y una baja carga animal. Además, en estos sitios, el desarrollo de comunidades de hierbas perennes es mayor. Es deseable mantener un desarrollo de los diferentes estratos leñosos para ofrecer cobertura al suelo y mejorar la oferta forrajera en el monte del noreste de la provincia de Río Negro.
\end{abstract}

[Palabras clave: ecosistemas semiáridos; estepa arbustiva; arbustificación; uso de la tierra]

\begin{abstract}
Aвstract. Land use and conservation of woody vegetation in the Northeast of Patagonia. Natural systems are often managed for production and provision of environmental services. The main use of semiarid ecosystems of Northeastern Patagonia is grazing by livestock in rangelands. Native vegetation is a shrubby-grassland steppe with isolated groups of trees. In this work we study the relationships between compositional and structural aspects of vegetation and the anthropic use of the Patagonian Monte. Communities were characterized by ecological parameters such as richness, cover, Shannon Wiener's diversity index $\left(\mathrm{H}^{\prime}\right)$ and Importance Value Index (IVI). Sites with similar covers of high and low strata and sites dominated by high shrubby strata presented higher values of Shannon index, greater cover and lower cattle stock. Besides, these sites had higher cover of perennial herbs. Presence of different woody strata provides more cover to the soil and enhances the forage availability in the Monte of the northeastern of Rio Negro.
\end{abstract}

[Keywords: semiarid lands, shrub steppe, encroachment, land use]

\section{INTRODUCCIÓN}

Los agroecosistemas son ecosistemas manejados para producir alimentos y fibras. Estos sistemas son sometidos a modificaciones frecuentes e intensas de sus componentes bióticos y abióticos (Soriano and Sala 1983). Además de los componentes físicos y biológicos, las características de estos ecosistemas están influidas por los factores sociales y económicos que afectan las decisiones de los productores en relación a qué producir y a qué tecnologías usar (Bilenca et al. 2009). Por ello, las formas de manejo de los rodeos, las cargas animales por unidad de superficie y las técnicas de manejo de la cobertura de la vegetación leñosa (i.e., clareo de monte, tala rasa, uso del fuego) para favorecer el desarrollo del estrato herbáceo, variarán de un período a otro y de una unidad productiva a otra y afectarán de forma

Editor asociado: Esteban Jobbágy diferencial los sistemas naturales involucrados (Kröpfl et al. 2007; Villagra et al. 2009).

El 41\% de los agroecosistemas mundiales están ubicados en zonas áridas y semiáridas, y en ellos se produce el $44 \%$ de las cosechas que se consumen a nivel global. Entre 10 y $20 \%$ de ecosistemas áridos y semiáridos del mundo experimentan procesos marcados de degradación (Abraham et al. 2011). En algunos casos, esta degradación se manifiesta por la creciente cobertura y dominancia de especies leñosas arbustivas (arbustificación); en otros casos, por la disminución y la pérdida de la cobertura total (Eldridge et al. 2011; GonzálezRoglich et al. 2015; Maestre et al. 2009). Estos procesos que alteran la dinámica natural de los sistemas afectados determinan una degradación creciente de la vegetación y se relacionan con factores de disturbio antrópicos o naturales, como el sobrepastoreo y la falta de 
manejo y rotación de los rodeos, o los incendios naturales (Eldridge et al. 2011; Eldridge and Soliveres 2015; Maestre et al. 2009).

En regiones áridas y semiáridas, el fuego actúa como modelador de la estructura y de la composición de la vegetación según la intensidad y la frecuencia del quemado (Bran et al. 2007; Kropfl et al. 2007; Rostagno et al. 2006). El fuego es una herramienta muy empleada para controlar la cobertura de especies leñosas y favorecer el rebrote y el establecimiento de especies herbáceas (Villagra et al. 2009). Estas prácticas apuntan a mejorar la receptividad ganadera de los sistemas y son muy frecuentes en el noreste de la Patagonia (Kropfl et al. 2007). La herbívora reduce la biomasa de los pastos y de las hierbas perennes, como así también su cobertura; de esta manera dejan nichos disponibles para que las especies leñosas los colonicen y ocupen (Bisigato and Bertiller 1997; Guevara et al. 2009). Además, el fuego actúa en sentido opuesto a la herbivoría, ya que favorece la disminución de la cobertura leñosa y mejora la disponibilidad forrajera (Peter et al. 2013).

En el este de la provincia de Río Negro, las actividades productivas predominantes son la ganadería extensiva de monte y la agricultura de secano (esta última ocupa una superficie creciente). La producción agropecuaria trae aparejada una serie de transformaciones profundas que afectan prácticamente todos los procesos ecológicos y los flujos de materiales y energía en los ecosistemas (Sala et al. 2000). Está muy aceptado que la ganadería extensiva, o ganadería de monte, es el uso del suelo mas compatible con la conservación de la diversidad biológica y de los suelos, en comparación con la agricultura extensiva. Sin embrago, si se compara la ganadería extensiva con la exclusión completa de las actividades pecuarias, la ganadería extensiva puede ser perjudicial, levemente neutra, o incluso, deseable para el manejo y conservación de los recursos naturales (Cingolani et al. 2008; McNaughton 1983, 1985).

De acuerdo con la intensidad en el uso del suelo, los sistemas de esta región varían entre una vegetación abierta de pastizal, con arbustos bajos y dispersos, y un sistema dominado por leñosas (Torres Robles et al. 2015). Por otra parte, los sitios que perdieron su cobertura vegetal a causa de fuegos intensos o desmontes, y que sufrieron fuertes procesos erosivos del suelo, difícilmente se recuperarán sin una intervención deliberada dirigida a restaurar las funciones ecosistémicas perdidas (Zeberio 2012). Por estos motivos, si se parte de la idea de que el uso ganadero con un adecuado manejo de los rodeos, una adecuada determinación de la carga animal y con un manejo de la cobertura vegetal, determinará un sistema más diverso y mejor conservado. En este sentido, consideramos que los sitios que presenten una mayor cobertura de los distintos estratos de la vegetación leñosa presentarán una mayor cobertura de vegetación herbácea perenne y, por ende, poseerán un mejor estado de conservación. El objetivo del presente trabajo es relacionar aspectos estructurales, composicionales y de uso antrópico de las comunidades del monte presentes en el este de la provincia de Río Negro para evaluar el estado de conservación de la vegetación.

\section{Materiales y MÉtodos}

\section{Área de estudio}

El trabajo se llevó a cabo en el este de la provincia de Río Negro, en un área situada entre los $40^{\circ} 23^{\prime} 44^{\prime \prime}-41^{\circ} 11^{\prime} 1^{\prime \prime} \mathrm{S}$ y los $62^{\circ} 34^{\prime} 16^{\prime \prime}$

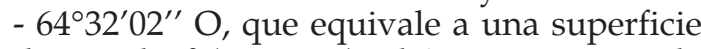
de 8813 km² (Figura 1). El área pertenece a la ecorregión denominada Monte de Llanuras y Mesetas (Morello et al. 2012) o Provincia Fitogeográfica del Monte (Cabrera 1971), que presenta una heterogeneidad ambiental amplia. El clima delárea de estudio es templado semiárido. La temperatura mínima media de julio es $7^{\circ} \mathrm{C}$, la máxima media de enero es 24 ${ }^{\circ} \mathrm{C}$ y la temperatura media anual es $14^{\circ} \mathrm{C}$. La precipitación media anual es de $270 \mathrm{~mm}$, con dos picos estacionales, uno en otoño y otro en primavera. La evapotranspiración media anual para la región es de $800 \mathrm{~mm}$ (Godagnone and Bran 2009). Los suelos predominantes son Paleargides y Haplargides típicos, con bajos porcentajes de materia orgánica $(<1.5 \%)$ y de nutrientes (Godagnone and Bran 2009).

La vegetación del área se corresponde con una estepa arbustiva media a alta, en la que suelen aparecer elementos arbóreos más o menos aislados y formando bosquecillos (León et al. 1998). La distribución espacial de la vegetación se observa sobre una matriz de suelo desnudo en la cual aparecen parches de vegetación dominados por especies leñosas que confieren protección contra la herbivoría y ofrecen mejores condiciones para el establecimiento de renovales (Aguiar and Sala 1998). La vegetación leñosa suele estar acompañada de un estrato herbáceo conformado por especies nativas de gramíneas 


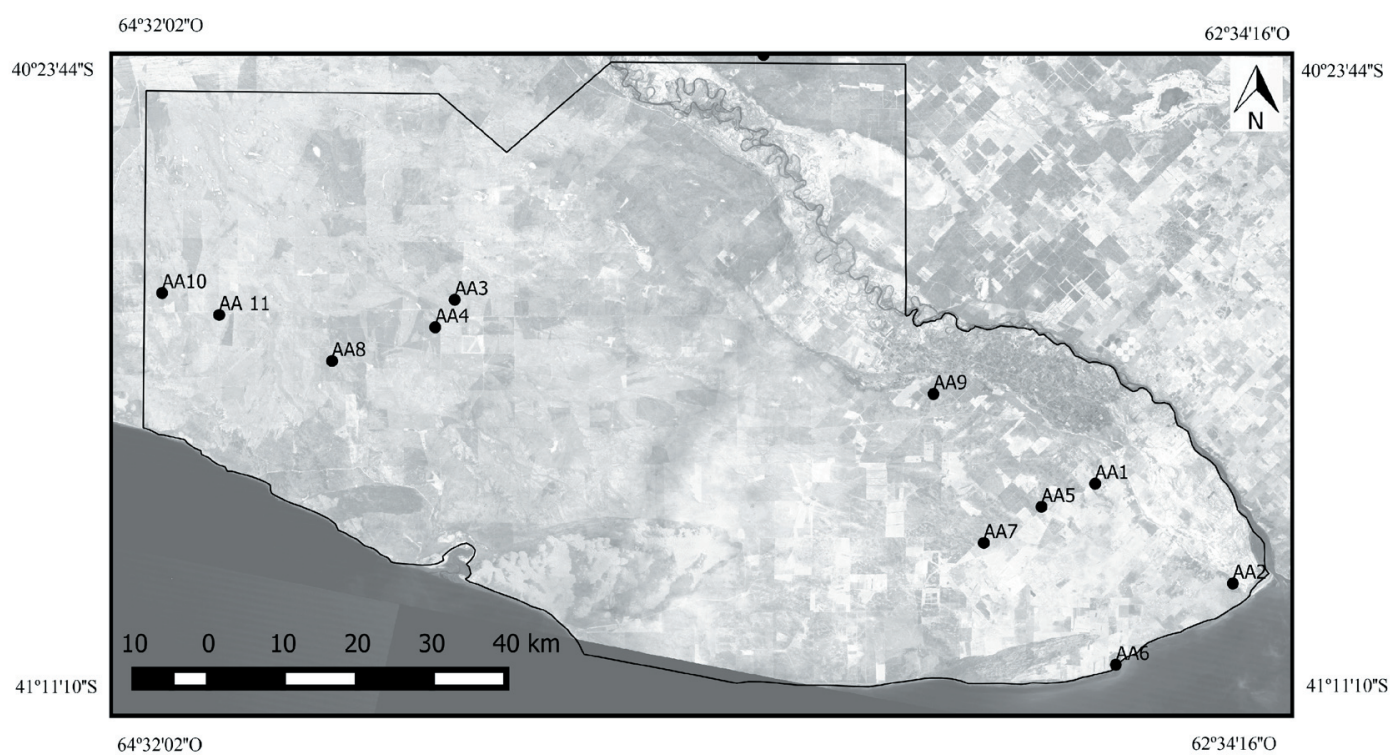

Figura 1. Ubicación geográfica de los sitios muestreados en el área de estudio. AA: Adolfo Alsina. Los números asociados indican el número de sitio.

Figure 1. Study area and location of sampling sites. AA: Adolfo Alsina. Associated numbers indicate site number.

y dicotiledóneas, muchas de ellas endémicas, que, de acuerdo con la historia de uso de las unidades productivas, estarán presentes en mayor o menor porcentaje, o incluso ausentes.

Las principales actividades productivas de la región son la ganadería extensiva de monte $\mathrm{y}$, en sectores desmontados, la agricultura extensiva. En los establecimientos dedicados a la ganadería es frecuente que se cultiven granos y forrajes. Se suelen realizar desmontes en franjas donde se siembran estos cultivos para suplementar la alimentación de los rodeos.

\section{Relevamiento de usos del suelo}

En el año 2015 se encuestó a los propietarios de las unidades productivas donde se realizaron los muestreos. Se indagó acerca de la actividad desarrollada en sus establecimientos entre los años 2012 y 2015, fecha en la que se realizaron los censos de vegetación. Se registró el uso dominante del suelo, es decir, si este era puramente ganadero o se efectuaban tareas de siembras intercaladas con el monte para favorecer la oferta de forraje (uso mixto). También se recabo información sobre la carga animal con la que se suelen poblar los campos de la región. Dado que en la zona hay presencia de ganado vacuno y ovino, se homogeneizó el dato transformando las existencias de ganado ovino a equivalente vaca (EV) (Bayer 2011). De acuerdo con el número de animales, la carga puede ser intensa $\left(>0.12 \mathrm{EV} \cdot \mathrm{ha}^{-1} \cdot \mathrm{año}^{-1}\right)$, media (0.12 EV.ha ${ }^{-1} \cdot$ año $\left.^{-1}-0.09 \mathrm{EV} / \mathrm{ha}^{-1} \cdot \mathrm{año}^{-1}\right)$ o liviana $\left(<0.09 \mathrm{EV} / \mathrm{ha}^{-1}\right.$.año $\left.{ }^{-1}\right)($ Kröpfl et al. 2015). Además, se obtuvo información acerca del tipo de manejo de los rodeos: rotación (movimiento de la hacienda por distintos sectores del predio) o año redondo (sin movimiento de la hacienda), la ocurrencia de incendios que afectaron sus propiedades. También se registró si realizan algún tipo de manejo de la cobertura leñosa para favorecer el rebrote de pastos y hierbas.

\section{Estructura y composición de la vegetación}

En un trabajo previo se describió la variación de la estructura y composición de diferentes localidades del noreste de la Patagonia (Torres Robles et al. 2015). En el presente trabajo se incluyen cinco de los sitios relevados y se amplía el muestreo a un total de 11 sitios en el departamento Adolfo Alsina, provincia de Río Negro. El criterio de selección de los sitios fue la variación de la cobertura de la vegetación con relación al uso del suelo de manera tal de incluir sitios sobrepastoreados, con evidencia de fuego y de manejo de la cobertura leñosa por cadeneo, rolado o desmonte en franjas. En este sentido, se empleó la interpretación de imágenes Landsat 8 OLI para identificar sitios.

El muestreo se realizó estratificado según las fisonomías halladas. En cada sitio de muestreo se establecieron 3 parcelas, de $100 \mathrm{~m}^{2}$ mediante un muestreo sistemático, 
separadas por una distancia mínima de 50 m. Para las especies leñosas se registró la presencia/ausencia de cada especie en cada parcela. La nomenclatura de las especies registrados sigue la flora argentina (Antón and Zuloaga 2014). Además, se registró la altura y se midió el ancho máximo de la copa y la medida perpendicular al mismo para calcular la cobertura como el área de un círculo con diámetro igual al promedio de las medidas de la copa (Mateucci and Colma 1982). En función de las alturas registradas se estimó la cobertura del estrato arbóreo y del estrato arbustivo alto $(>1 \mathrm{~m})$ y bajo $(<1 \mathrm{~m})$. A partir del número total de especies leñosas registradas en cada sitio se estimó la riqueza de especies por estrato de la vegetación (Mateucci and Colma 1982). Se calculó la frecuencia de especies leñosas por parcela para calcular el índice de diversidad de Shannon-Wiener $\left(\mathrm{H}^{\prime}\right)$ de cada sitio, que es una medida de la abundancia de especies presentes en cada uno de los sitios analizados (Shannon and Wiener 1949).

Con la finalidad de establecer una relación entre la cobertura de la vegetación leñosa y herbácea, para cada parcela donde se relevó la estructura de leñosas, se estimó la cobertura de las especies de pastos perennes mediante el método de intercepción lineal a lo largo de una transecta de $10 \mathrm{~m}$ de longitud (Kangas and Maltamo 2006; Mateucci and Colma 1982).

Para cada especie presente, En cada sitio se estimó el índice de valor de importancia (IVI), que es un descriptor de la importancia ecológica de una determinada especie dentro de una comunidad. Resulta de la sumatoria de la frecuencia, la abundancia y la densidad o cobertura de cada especie, por lo que es un predictor más robusto de la importancia de cada especie que sus parámetros evaluados individualmente (Mateucci and Colma 1982; Mostacedo and Fredericksen 2000). Se estimó y se relativizó la abundancia, la frecuencia y la dominancia (esta última en función de la cobertura de las diferentes especies leñosas censadas). Con estos datos se estimó el IVI según la fórmula:

$\mathrm{IVI}=100 * \mathrm{Aa}_{\mathrm{i}} / \mathrm{Aa}_{\mathrm{t}}+100 * \mathrm{Fa}_{\mathrm{i}} / \mathrm{Fa}_{\mathrm{t}}+100$ * $\mathrm{Da}_{\mathrm{i}} / \mathrm{Da}_{\mathrm{t}}$

Donde: Aa: abundancia absoluta de la especie (i)

Aa: suma de la abundancia de todas las especies $(t)$
$\mathrm{Fa}_{\mathrm{i}}$ : frecuencia absoluta de la especie (i)

$\mathrm{Fa}_{\mathrm{t}}$ : suma de las frecuencias de todas las especies ( $t$ )

$\mathrm{Da}_{\mathrm{i}}$ : cobertura absoluta de la especie (i)

$\mathrm{Da}_{\mathrm{t}}$ : suma de la cobertura de todas las especies $(\mathrm{t})$.

El IVI puede fluctuar entre 0 y 300\%. Al dividir el valor de importancia por 3 se obtiene una cifra que fluctúa entre 0 y $100 \%$. Este valor se conoce como el porcentaje de importancia, que constituye un estimador global de la importancia de una especie en una comunidad determinada (Acosta et al. 2006; Mateucci and Colma 1982).

\section{Análisis de los datos}

Los datos de riqueza de especies leñosas, cobertura de especies leñosas por estratos, diversidad de especies leñosas y cobertura de especies herbáceas en los distintos sitios no cumplían con el supuesto de normalidad y homocedasticidad. Por esta razón se empleó un análisis de la varianza no paramétrico. Se usó la prueba de Kruskal-Wallis con comparaciones de a pares, con un nivel de significancia de $5 \%$.

Para estimar en qué medida las variables de vegetación (i.e., cobertura de especies herbáceas, cobertura de especies leñosas por estrato, diversidad de especies leñosas, riqueza de especies arbóreas, arbustivas de los estratos alto y bajo) y la información sobre el uso del suelo (i.e., uso dominante, manejo de rodeos, carga animal, ocurrencia de incendios y manejo de la cobertura) se relacionan entre ellas, se utilizó un análisis de componentes principales (ACP). Las variables de uso del suelo cualitativas fueron transformadas a variables binarias. De esta manera a los sitos que presentaron uso mixto se le asignó la categoría 1; a los que sólo presentaron uso ganadero, la categoría 0; a los que presentaron rotación de los rodeos, la categoría 1, y a los que presentan pastoreo continuo, la categoría 0 . La ocurrencia de incendios se categorizó como categoría 1 y la ausencia de incendios como categoría 0 . Al manejo de la cobertura se le asignó la categoría 1 y a la ausencia de la misma, categoría 0. El software usado en todos los análisis fue InfoStat (Di Rienzo et al. 2008). 


\section{Resultados}

\section{Relevamiento de usos del suelo}

El uso dominante en los diferentes sitios analizados es la ganadería extensiva por sobre el uso mixto de la tierra (Tabla 1). El manejo rotativo de los rodeos se realiza en $45 \%$ de los sitios encuestados. En el 64\% de los establecimientos la carga animal es intensa, y en el 36\% el pastoreo fue liviano (Tabla 1). Los incendios registrados, la mayoría de ellos en la temporada estival de los años 2000-01, afectaron al 55\% de las unidades evaluadas. Sólo en tres de los 11 sitios se realiza manejo de la cobertura leñosa (Tabla 1).

\section{Estructura y composición de la comunidad}

Se encontraron dos especies arbóreas y 22 especies arbustivas en el área de estudio (Tabla 2; Tabla 4). La riqueza de especies de árboles presenta diferencias altamente significativas $(P<0.01)$ entre los sitios, registrándose en cuatro de los 11 sitios analizados (Tabla 2). Sólo Geoffroea decorticans resultó la especie de mayor IVI en uno de estos sitios (AA1); en el resto de los sitios, las especies con mayor IVI son arbustivas, y las arbóreas aparecen con menor importancia (Tabla 4).
Las diferencias entre la cobertura del estrato arbustivo, tanto alto como bajo, muestra diferencias significativas entre sitios $(P<0.05)$. Los sitios que presentan una mayor cobertura del estrato arbustivo alto tienden a tener una menor cobertura del estrato arbustivo bajo (Tabla 3).

Las riquezas de los estratos arbustivos alto y bajo también presentaron diferencias significativas entre sitios $(P<0.05)$. Los sitios que presentan mayor riqueza de arbustos del estrato alto $(>7)$ presentan también alta riqueza de arbustos del estrato bajo (>6) (Tabla 2). Las especies que presentaron mayor IVI en el estrato arbustivo alto son Lycium chilense, Larrea divaricata, Chuquiraga erinacea, Condalia microphylla y Schinus johnstonii. Las especies con mayor IVI del estrato arbustivo bajo son Senna aphylla, Prosopis alpataco, Acantholippia seriphyoides, Ephedra triandra y Baccharis ulicina. También en este estrato aparecen con un alto IVI especies como Larrea divaricata, Condalia microphylla y Schinus johnstonii, pero con un menor grado de desarrollo. Aquellos sitios con una riqueza intermedia (5-7) de arbustos del estrato alto presentan una riqueza intermedia de arbustos del estrato bajo (2-4) (Tabla 2). Un sitio (AA 1) presentó una baja riqueza

Tabla 1. Variables de uso del suelo registradas en los diferentes establecimientos en los que se relevó la estructura y composición de la vegetación. AA: Adolfo Alsina. Los números asociados indican el número de sitio.

Table 1. Land use variables in each sampled point. AA: Adolfo Alsina. Associated numbers indicate site number.

\begin{tabular}{cllccc}
\hline Sitio & Uso dominante & Manejo de rodeos & $\begin{array}{c}\text { Carga animal/ } \\
\text { ha }\end{array}$ & $\begin{array}{c}\text { Ocurrencia de } \\
\text { incendios }\end{array}$ & $\begin{array}{c}\text { Manejo de la } \\
\text { cobertura }\end{array}$ \\
\hline AA1 & Ganadera extensiva & Rotación & 0.30 & No & No \\
AA2 & Ganadera extensiva & Rotación & 0.17 & $\mathrm{No}$ & No \\
AA3 & Ganadera extensiva & Rotación & 0.29 & $\mathrm{Si}$ & No \\
AA4 & Ganadera extensiva & Rotación & 0.04 & $\mathrm{Si}$ & No \\
AA5 & Ganadería mixta & Pastoreo continuo & 0.06 & $\mathrm{No}$ & No \\
AA6 & Ganadería mixta & Pastoreo continuo & 0.05 & $\mathrm{Si}$ & $\mathrm{So}$ \\
AA7 & Ganadera extensiva & Pastoreo continuo & 0.18 & $\mathrm{Si}$ & $\mathrm{Si}$ \\
AA8 & Ganadera extensiva & Pastoreo continuo & 0.23 & $\mathrm{No}$ & $\mathrm{No}$ \\
AA9 & Ganadera extensiva & Pastoreo continuo & 0.15 & $\mathrm{Si}$ & $\mathrm{Si}$ \\
AA10 & Ganadería mixta & Pastoreo continuo & 0.20 & $\mathrm{Si}$ & $\mathrm{Si}$ \\
AA11 & Ganadería mixta & Rotación & 0.08 & & \\
\hline
\end{tabular}

Tabla 2. Riqueza de especies arbóreas, especies del estrato arbustivo alto y del estrato arbustivo bajo e índice de Shannon- Wiener $\left(\mathrm{H}^{\prime}\right)$ calculado para las especies leñosas presentes en cada sitio. Medias con una letra común no son significativamente diferentes $(P<0.05)$ para los distintos valores de riqueza de los estratos arbóreos y arbustivos alto y bajo. AA: Adolfo Alsina. Los números asociados indican el número de sitio.

Table 2. Species richness in arboreal strata, high shrub strata and low shrub strata, and Shannon and Wiener index calculated for woody vegetation $\left(\mathrm{H}^{\prime}\right.$ leñosas). Mean richness values for each stratum with the same letter are not significantly different $(P<0.05)$. AA: Adolfo Alsina. Associated number indicate site number.

\begin{tabular}{lcccccccccccc}
\hline & AA1 & AA10 & AA5 & AA2 & AA9 & AA11 & AA6 & AA7 & AA4 & AA3 & AA8 & $P$ \\
\hline Riqueza de árboles & $1 \mathrm{~b}$ & $1 \mathrm{~b}$ & $1 \mathrm{~b}$ & $0 \mathrm{a}$ & $1 \mathrm{~b}$ & $0 \mathrm{a}$ & $0 \mathrm{a}$ & $0 \mathrm{a}$ & $0 \mathrm{a}$ & $0 \mathrm{a}$ & $0 \mathrm{a}$ & 0.0039 \\
Riqueza de arbustos altos & $\mathrm{a}$ & $7 \mathrm{~b}$ & $6 \mathrm{ab}$ & $5 \mathrm{ab}$ & $7 \mathrm{~b}$ & $7 \mathrm{~b}$ & $7 \mathrm{~b}$ & $10 \mathrm{c}$ & $12 \mathrm{c}$ & $9 \mathrm{c}$ & $6 \mathrm{ab}$ & 0.0331 \\
Riqueza de arbustos bajos & $0 \mathrm{a}$ & $2 \mathrm{abc}$ & $4 \mathrm{abcde}$ & $4 \mathrm{abcde}$ & $4 \mathrm{abcde}$ & $1 \mathrm{ab}$ & $4 \mathrm{abcde}$ & $6 \mathrm{cde}$ & $10 \mathrm{e}$ & $7 \mathrm{cde}$ & $9 \mathrm{de}$ & 0.0123 \\
H' leñosas & $0.8 \mathrm{a}$ & $1.7 \mathrm{abcd}$ & $1.7 \mathrm{abcd}$ & $1.4 \mathrm{ab}$ & $1.6 \mathrm{abc}$ & $1.3 \mathrm{ab}$ & $1.6 \mathrm{abc}$ & $2 \mathrm{bcd}$ & $2.3 \mathrm{~d}$ & $2 \mathrm{~cd}$ & $1.8 \mathrm{bcd}$ & 0.0169 \\
\hline
\end{tabular}


Tabla 3. Porcentaje de cobertura media estimada para el estrato arbustivo alto, estrato arbustivo bajo, estrato arbóreo y estrato herbáceo para cada sitio. Medias con una letra común no son significativamente diferentes $(P<0.05)$ para los distintos niveles de cobertura de los estratos arbustivo alto y bajo. AA: Adolfo Alsina. Los números asociados indican el número de sitio. D.E.: desvío estándar.

Table 3. Mean cover percentage estimated in arboreal strata, high shrub strata, low shrub strata and herb strata for each site. Mean values in high and low shrub coverage with a common letter are not different $(P<0.05)$. AA: Adolfo Alsina. Associated number indicate site number. D.E.: standard deviation.

\begin{tabular}{ccccccccc}
\hline & \multicolumn{2}{c}{ Arbustivo alto } & \multicolumn{2}{c}{ Arbustivo bajo } & \multicolumn{2}{c}{ Arbóreo } & \multicolumn{2}{c}{ Herbáceo } \\
Sitio & Medias & D.E. & Medias & D.E. & Medias & D.E. & Medias & D.E. \\
\hline AA1 & $19.2 \mathrm{abc}$ & 6.9 & $0 \mathrm{a}$ & 0 & $46.3 \mathrm{~b}$ & 12.7 & $3 \mathrm{ab}$ & 1.7 \\
AA2 & $44.4 \mathrm{~d}$ & 18.1 & $7.1 \mathrm{abc}$ & 1.5 & $0 \mathrm{a}$ & 0 & $0 \mathrm{a}$ & 0 \\
AA3 & $24.4 \mathrm{abcd}$ & 4.8 & $12 \mathrm{abc}$ & 10.5 & $0 \mathrm{a}$ & 0 & $34.7 \mathrm{~cd}$ & 24 \\
AA4 & $26.8 \mathrm{abcd}$ & 8.6 & $21.4 \mathrm{c}$ & 9.1 & $0 \mathrm{a}$ & 0 & $10.7 \mathrm{abc}$ & 9.3 \\
AA5 & $18.8 \mathrm{ab}$ & 3.5 & $10.5 \mathrm{abc}$ & 5.9 & $0.3 \mathrm{ab}$ & 0.5 & $64.7 \mathrm{~d}$ & 23.5 \\
AA6 & $32.9 \mathrm{bcd}$ & 9.2 & $14 \mathrm{bc}$ & 6.9 & $0 \mathrm{a}$ & 0 & $7 \mathrm{abc}$ & 6.9 \\
AA7 & $28.1 \mathrm{abcd}$ & 13.9 & $23.7 \mathrm{c}$ & 2.3 & $0 \mathrm{a}$ & 0 & $10.3 \mathrm{abc}$ & 2.1 \\
AA8 & $10 \mathrm{a}$ & 5 & $28 \mathrm{c}$ & 13.1 & $0 \mathrm{a}$ & 0 & $2.3 \mathrm{a}$ & 1.5 \\
AA9 & $36.4 \mathrm{~cd}$ & 11.1 & $11.4 \mathrm{abc}$ & 11.5 & $1.8 \mathrm{a}$ & 0 & $53.7 \mathrm{~d}$ & 4.7 \\
AA10 & $26.8 \mathrm{bcd}$ & 3.6 & $7.2 \mathrm{ab}$ & 12.5 & $10.1 \mathrm{ab}$ & 9.7 & $28 \mathrm{bcd}$ & 6.1 \\
AA11 & $40.2 \mathrm{~cd}$ & 16 & $3.7 \mathrm{ab}$ & 3.8 & $0 \mathrm{a}$ & 0 & $35.3 \mathrm{~cd}$ & 3.1 \\
$P$ & 0.0298 & \multicolumn{7}{c}{0.0317} \\
\hline
\end{tabular}

Tabla 4. Índice de valor de importancia (IVI), expresado en \% de valor de importancia para cada especie presente en los diferentes sitios. AA: Adolfo Alsina. Los números asociados indican el número de sitio. *Árbol; **Arbusto.

Table 4. Importance value index (IVI), expressed in percentage for each species present in different sites. AA: Adolfo Alsina. Associated number indicate site number. ${ }^{*}$ Tree; ${ }^{* *}$ Shrub.

\begin{tabular}{|c|c|c|c|c|c|c|c|c|c|c|c|}
\hline & AA1 & AA10 & AA5 & AA2 & AA11 & AA9 & AA6 & AA7 & AA4 & AA3 & AA 8 \\
\hline Geoffroea decorticans* & 62.8 & 15.3 & 3.2 & & & & & & & & \\
\hline Prosopis flexuosa* & & & & & & 6.6 & & & & & \\
\hline Lycium chilense $^{* *}$ & 11.3 & 7.3 & 5.3 & 19.1 & 6.5 & 7.7 & 14.4 & 11.5 & 9 & 5.7 & 4 \\
\hline Larrea divaricata** & 5.3 & 28.5 & 13.8 & 44.9 & 21.5 & 16.4 & & 2.3 & 12.1 & 14.7 & \\
\hline Chuquiraga erinacea** & 1.7 & 9 & 32.2 & 5.8 & & 29.4 & 25.1 & 32.3 & 14.2 & 23.7 & 29 \\
\hline Condalia microphylla** & 10.2 & 1.9 & 11.3 & 10.8 & 13.4 & 14.9 & 19.4 & 5.3 & 10.6 & 7.1 & 8.8 \\
\hline Schinus johnstonii ${ }^{* *}$ & & 6.7 & 14 & & 4.5 & 9.2 & 8.8 & 8.6 & 5.3 & 10.9 & 7.7 \\
\hline Lycium tenuispinosum ${ }^{* *}$ & 8.6 & & 6.3 & & & 4.2 & 9.2 & 4.5 & & 2.2 & 0.8 \\
\hline Senna aphylla** & & 6.8 & & & 6.6 & & & 4.8 & 9.4 & 6.9 & 6.8 \\
\hline Larrea cuneifolia ${ }^{* *}$ & & 6.2 & & & 9.2 & & & & & & \\
\hline Prosopis alpataco ${ }^{* *}$ & & 5.8 & & & 22.7 & 2.1 & & 6.3 & 4.3 & 4.9 & 5.3 \\
\hline Acantholippia seriphioides** & & 5.7 & 7 & 9.8 & & 3.2 & 1.2 & & 3.9 & 6.2 & 1.9 \\
\hline Junellia seriphioides ${ }^{* *}$ & & 2.4 & & & & & & & & & \\
\hline Monttea aphylla** & & 2.4 & & & 2.6 & 1.8 & & 1.7 & 1 & 4.3 & \\
\hline Ephedra triandra** & & 1.9 & & & 1.9 & & & 4.8 & 4.7 & 0.7 & 3.2 \\
\hline Brachyclados lycioides ${ }^{* *}$ & & & 4.1 & 2.9 & & & & & 1.2 & 4 & 2.4 \\
\hline Baccharis crispa ${ }^{* *}$ & & & 1.6 & & & & & 1.7 & & & \\
\hline Baccharis ulicina ${ }^{* *}$ & & & 1.3 & & & 3.4 & 1.2 & 6.7 & 7.2 & 2.7 & 8.7 \\
\hline Aloysia gratissima** & & & & & & 1.1 & 1.2 & & & & \\
\hline Bougainvillea spinosa** & & & & & & & & & 1 & & \\
\hline Bredemeyera microphylla** & & & & & & & & 0.8 & 1 & 1.2 & 5.2 \\
\hline Cereus aethiops & & & & 1.8 & & & & & 1 & & \\
\hline Chuquiraga avellanedae & & & & & & & & & 9.3 & & \\
\hline Chuquiraga rosulata** & & & & & 8.2 & & & & & & \\
\hline Cyclolepis genistoides** & & & & & & & 13.1 & 1.4 & 1 & 2.3 & 2.7 \\
\hline Ephedra ochreata** & & & & & & & 6.2 & & & 1.6 & \\
\hline Grindelia chiloensis ${ }^{* *}$ & & & & & & & & & & & 0.8 \\
\hline Lycium gillesianum ${ }^{* *}$ & & & & 5 & & & & & & & \\
\hline Margyricarpus pinnatus** & & & & & & & & & & & 4 \\
\hline Prosopidastrum globosum ${ }^{* *}$ & & & & & 2.7 & & & 7.3 & 3.5 & 0.9 & 8.7 \\
\hline Total & 100 & 100 & 100 & 100 & 100 & 100 & 100 & 100 & 100 & 100 & 100 \\
\hline
\end{tabular}


de especies del estrato arbustivo alto sin la presencia del estrato arbustivo bajo (Tabla 2).

El índice de diversidad $\mathrm{H}^{\prime}$, estimado para la vegetación leñosa, muestra diferencias estadísticamente significativas $(P<0.05)$ entre sitios (Tabla 2). El valor de $\mathrm{H}^{\prime}$ más bajo $\left(\mathrm{H}^{\prime}<1.3\right)$ se encontró en el sitio donde predominó el estrato arbóreo, con G. decorticans como la especie con mayor IVI (Tabla 4). Los valores de $\mathrm{H}^{\prime}$ intermedios (1.3-1.8) y altos $\left(\mathrm{H}^{\prime}>1.8\right)$ se encontraron en sitios donde predomina la cobertura del estrato arbustivo alto por sobre el arbustivo bajo o en situaciones en las que ambos estratos presentaron similares porcentajes de cobertura (Tabla 3). El sitio en el que predominó la cobertura del estrato bajo por sobre la cobertura del estrato alto presentó también un valor alto de $\mathrm{H}^{\prime}$ (Tabla 2).

La cobertura del estrato conformado por especies herbáceas de ciclo de vida perenne presenta diferencias altamente significativas
$(P<0.01)$, con valores que van desde 0 , es decir la ausencia total de especies de hierbas perennes, hasta sitios donde la cobertura de este estrato es superior al 50\%. Los sitios que presentaron mayor cobertura de especies herbáceas fueron aquellos que presentaron mayor cobertura del estrato arbustivo alto y riqueza de especies arbóreas (Tabla 3; Figura 2). En el sitio AA1, donde predominó el estrato arbóreo, la presencia de especies herbáceas fue casi nula (Tabla 3).

\section{Relación entre la estructura de la vegetación y el uso del suelo}

El primer eje de ordenamiento de los sitios por variables estructurales y de uso reflejó un cambio en la fisonomía de la vegetación y en el uso del suelo, desde el extremo negativo (donde los sitios presentaron mayor cobertura del estrato arbóreo, rotación de los rodeos y mayor carga animal), hacia el extremo positivo [con sitios con mayor cobertura del estrato arbustivo bajo (20-28\%), mayor diversidad de

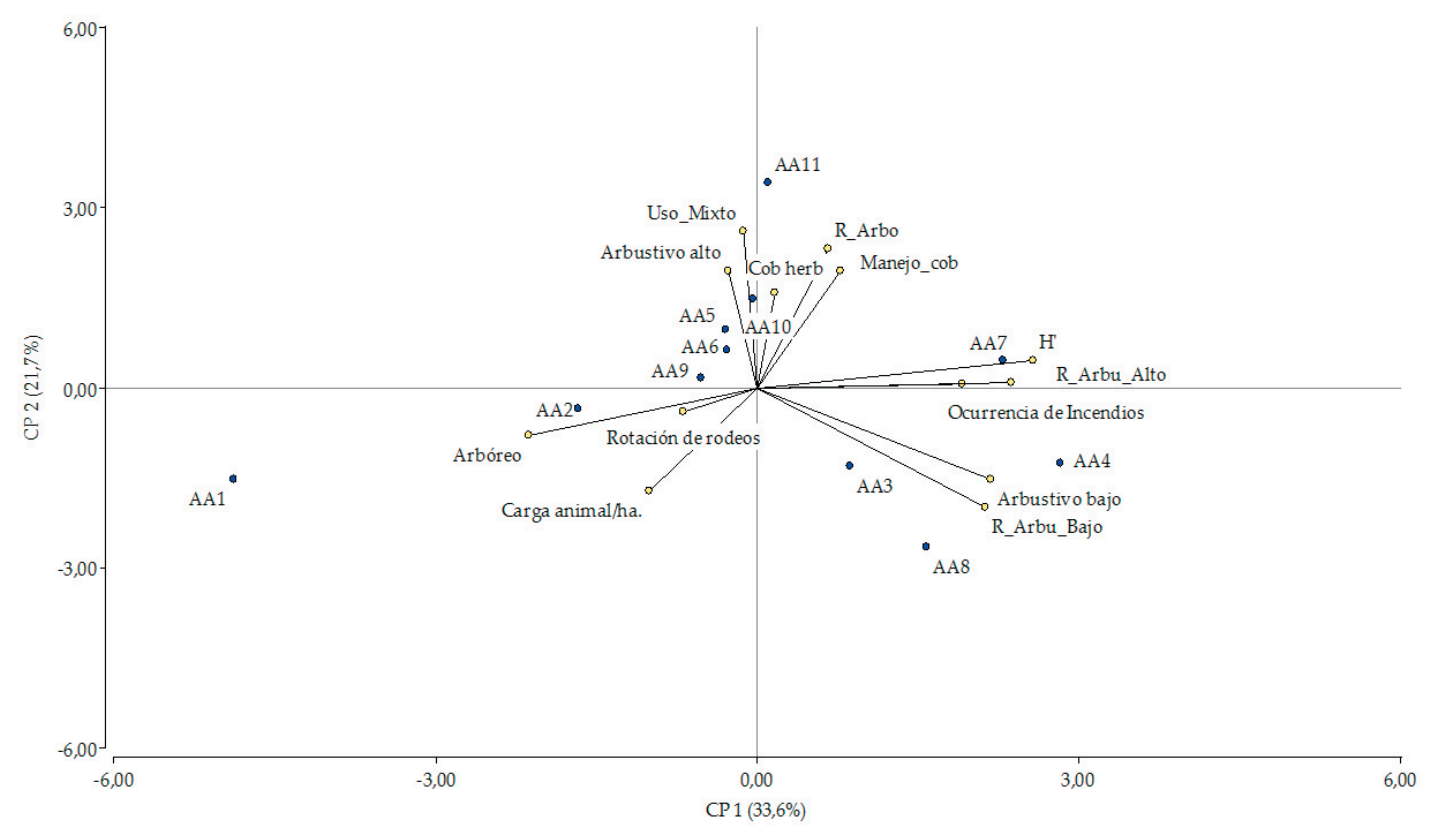

Figura 2. Ordenamiento de los sitios sobre la base de la frecuencia de las variables estructurales (arbóreo; cobertura estrato arbóreo; arbustivo alto; cobertura del estrato arbustivo alto; arbustivo bajo; cobertura del estrato arbustivo bajo; Cob_herb: cobertura del estrato herbáceo), de composición ( $\mathrm{H}^{\prime}$ : índice de Shannon-Wiener estimado para especies leñosas; R_Arbo: riqueza de especies arbóreas; R_Arbu_alto: riqueza de especies arbustivas del estrato alto; R_Arbu_Baja: riqueza de especies leñosas del estrato arbustivo bajo) y de uso (rotación de rodeos; ocurrencia de incendios; carga animal/ha; mayor carga animal [estimada en equivalentes vaca por hectárea]; uso mixto). CP: componente principal (se indica entre paréntesis el valor de varianza explicada por cada componente). AA: Adolfo Alsina. Los números asociados indican el número de sitio.

Figure 2. Sites ordination based on structural variables (cobertura estrato arbóreo: arboreal strata coverage; cobertura estrato arbustivo alto: high shrub coverage strata; cobertura estrato arbustivo bajo: low shrub coverage strata; cobertura estrato herbáceo: herb coverage strata), composition ( $\mathrm{H}^{\prime}$ : Shannon-Wiener index [estimated for shrub vegetation] and land use (rotación de rodeos: rodeo rotation; ocurrencia de incendios: fire events; carga animal/ha: animal load; uso mixto: mixed land use; manejo de la cobertura: cover management). CP: principal component (the variance value explained for each component is indicated between parenthesis). AA: Adolfo Alsina. Associated number indicate site number. 
especies leñosas, mayor riqueza de especies del estrato arbustivo alto y mayor ocurrencia de incendios (Figura 2). El CP 2 también reflejó una variación en la mayor cobertura del estrato arbustivo alto y uso del suelo, con ganadería mixta y manejo de la cobertura en el extremo positivo hacia situaciones con mayor cobertura del estrato arbustivo bajo hacia el extremo negativo, con uso del suelo con ganadería extensiva con una alta carga animal/ha. (Figura 2).

\section{Discusión}

De los sitios analizados, el pastoreo intenso se vinculó tanto a sitios donde predominó la cobertura leñosa como en los que predominó la cobertura del estrato arbustivo bajo, y presentaron una baja cobertura del estrato herbáceo perenne. Eldridge et al. (2011), Eldridge y Soliveres (2015) y Maestre et al. (2009) plantean que el pastoreo intenso genera un aumento de la cobertura arbustiva, lo que indica un grado de deterioro alto y una pérdida de productividad del sistema. En el sitio con mayor cobertura de árboles, la especie de mayor valor de importancia fue G. decorticans. Además, este sitio presentó los valores más bajos de diversidad $\mathrm{H}^{\prime}$ de especies leñosas y una ausencia de cobertura herbácea. En los sitios en los que predominó el estrato arbustivo bajo, las especies de mayor importancia fueron Senna aphylla, Prosopis alpataco, Acantholippia seriphyoides, Ephedra triandra y Baccharis ulicina. La diversidad $\mathrm{H}^{\prime}$ de leñosas fue alta, vinculada a la ocurrencia de incendios. Algunas de las especies que registraron un alto IVI en el estrato arbustivo alto (e.g., Larrea divaricata, Condalia microphylla y Schinus johnstonii) presentaron también un alto IVI como parte del estrato arbustivo bajo. Esto indica que estas especies serían afectadas, por un lado, por la lenta tasa de crecimiento que poseen las especies leñosas nativas y, por otro, por el disturbio causado por el ganado doméstico, que impide su establecimiento y desarrollo. El fuego podría haber tenido un papel importante en la fisonomía de estos sitios, al dejar los suelos desprovistos de cobertura vegetal y al retardar su recuperación.

En ausencia de pastos y hierbas, las especies leñosas no encuentran interferencia para colonizar espacios libres y los ecosistemas naturales ingresan en un ciclo de arbustificación, pérdida de valor de uso cómo sistemas ganaderos y son más susceptibles a incendios de mayor intensidad y dimensión
(Ward et al. 2013). Una situación de este tipo es la observada por Bran et al. (2007), en la que, según la severidad del quemado, el ecosistema tardará más o menos tiempo en recuperarse y, al hacerlo, comenzará con la aparición de individuos de las especies leñosas de menor porte y que no ofrecen condiciones favorables en el establecimiento de nuevos individuos. Además, debido a la pérdida de fertilidad de los suelos de áreas quemadas (en particular, la pérdida de C orgánico) se dificultará la colonización de esos sitios por especies vegetales (Gaitán et al. 2007).

Tanto en sitios donde predomina el estrato arbustivo alto sobre el estrato arbustivo bajo y en los que se registró la presencia de árboles, pero con mayor desarrollo del estrato arbustivo alto, presentaron valores intermedios de $\mathrm{H}^{\prime}$ y mayor porcentaje de cobertura de especies herbáceas perennes. Las especies del estrato arbustivo alto de mayor importancia son Lycium chilense, Larrea divaricata, Chuquiraga erinacea, Condalia microphylla y Schinus johnstonii. En estos sitios, el uso del suelo dominante es mixto, con manejo de la cobertura leñosa y baja carga animal por hectárea. El uso mixto de la tierra es una práctica que reduciría la presión de pastoreo en épocas claves del año, ya que permitiría a los individuos del estrato herbáceo recuperar la biomasa removida por los rumiantes y generar estructuras reproductivas. Además, la mayor cobertura de este estrato está relacionada con un desarrollo de los estratos arbustivo alto y bajo, lo que daría mayor cantidad de micrositios disponibles para el establecimiento y desarrollo de especies herbáceas (Maestre et al. 2001). Archer (1994) y Kröpfl et al. (2015) realizaron observaciones similares, en las que señalan que los pastos pueden reducir la emergencia, el crecimiento y la supervivencia de plántulas leñosas, pero que esa reducción competitiva no sería suficiente para causar la mortalidad o la exclusión completa de los arbustos del sistema. Este efecto indica que sería deseable mantener una cobertura media o baja de los estratos leñosos para favorecer la presencia de especies herbáceas, que en conjunción con manejos adecuados de los rodeos redundarían en un sistema más diverso y productivo (Busso et al. 2016; Giorgetti et al. 2006).

El uso ganadero con un adecuado manejo de los rodeos, una adecuada determinación de la carga animal y con un manejo de la cobertura vegetal determinará un sistema más diverso 
y mejor conservado, ya que los sitios que tuvieron este tipo de manejo presentaron coberturas similares del estrato arbustivo alto y bajo o mayor cobertura del estrato arbustivo alto, exhibieron una mayor cobertura vegetal herbácea perenne y, por ende, un mejor estado de conservación.

\section{Conclusiones}

Tanto los sitios con mayor desarrollo del estrato arbustivo bajo como el sitio con mayor desarrollo del estrato arbóreo tendrían menor valor de conservación. Los sitios que presentaron una mayor cobertura y riqueza del estrato arbustivo bajo se caracterizaron por la presencia de Senna aphylla, Prosopis alpataco, Acantholippia seriphyoides, Ephedra triandra y Baccharis ulicina. Presentaron una baja cobertura de especies herbáceas perennes y una alta carga ganadera, y se vincularon con la ocurrencia de incendios. El único sitio donde dominó la cobertura del estrato arbóreo (Geoffroea decorticans) presentó un uso más intensivo, con alta carga ganadera y sin rotación ni manejo de la cobertura vegetal, los valores más bajos de riqueza y diversidad y un estrato herbáceo perenne ausente.
Entre los sitios que consideramos, los que presentan mayores valores deconservación son aquellos con desarrollo del estrato arbustivo alto, bajo y herbáceo. En estos sitios las especies arbóreas fueron Geoffroea decorticans y Prosopis flexuosa y las especies arbustivas del estrato alto de mayor importancia fueron Lycium chilense, Larrea divaricata, Chuquiraga erinacea, Condalia microphylla y Schinus johnstonii. En estos sitios se registró un uso de la tierra mixto, un manejo de la cobertura leñosa y una baja carga animal. Consideramos necesario continuar con estudios de la dinámica de la vegetación leñosa y profundizar en el estudio del manejo de la cobertura de la misma para mejorar las condiciones ecológicas y productivas del monte del noreste rionegrino. En este sentido, sería deseable integrar aquellos sitios que sean identificados con valores de conservación bajos a proyectos de restauración o rehabilitación ecológica.

Agradecimientos. El trabajo fue financiado por la Universidad Nacional de Río Negro (PI 40-C-355) y por el Ministerio Nacional de Ciencia y Técnica (PICTO 2010/0219). Agradecemos a los revisores anónimos por sus sugerencias y comentarios que permitieron mejorar el manuscrito.

\section{REFERENCIAS}

Abraham, M. E., M. L. Corso, and P. Maccagno. 2011. Capitulo 1. Tierras secas y desertificación en Argentina., in: Evaluación de La Desertificación En Argentina. Resultados Del Proyecto LADA/FAO. Pp. 11-64.

Anton, A. M., and F. O. Zuloaga. 2014. Flora Argentina. Flora vascular de la República Argentina. URL: www.floraar gentina.edu.ar [acceso: noviembre de 2016].

Acosta, V., P. Araujo, and M. Iturre. 2006. Caracteres Estructurales de las Masasa. Serie didáctica N²2. FCF- UNSE. Pp. 35.

Aguiar, M., and O. Sala. 1998. Interactions among grasses, shrubs, and herbivores in Patagonian grass-shrub steppes. Ecología Austral 8:201-210.

Archer, S. 1994. Woody plant encroachment into southwestern grasslands and savannas: rates, patterns and proximate causes. Pp. 13-64 in Ecological Implications of Livestock Herbivory in the West.

Bayer, W. 2011. Equivalente oveja, oveja patagónica y equivalente vaca. Sitio Argentino de Producción animal. URL: www.produccion-animal.com.ar/produccionaovina.

Bilenca, D., M. Codesido, C. González Fisher, and L. Pérez Carusi. 2009. Impactos de la diversidad agropecuaria sobre la biodiversidad en la ecorregión pampeana: impactos de la expansión agrícola y de la intensificación de la agricultura y la ganadería de campo, con algunas recomendaciones de manejo para su mitigación. INTA.

Bisigato, A. J., and M. B. Bertiller. 1997. Grazing effects on patchy dryland vegetation in northern Patagonia. Journal of Arid Environments 36:639-653.

Bran, D. E., G. A. Cecchi, J. J. Gaitán, J. A. Ayesa, and C. R. López. 2007. Efecto de la severidad de quemado sobre la regeneración de la vegetación en el Monte Austral. Ecología Austral 17:123-131.

Busso, C. A., O. A. Montenegro, Y. A. Torres, H. D. Giorgetti, and G. D. Rodríguez. 2016. Aboveground net primary productivity and cover of vegetation exposed to various disturbances in arid Argentina. Applied Ecology and Environmental Research 14:51-75.

Cabrera, A. L. 1971. Fitogeografía de la Republica Argentina. Boletín de la Sociedad Argentina de Botánica 14:1-50.

Cingolani, A. M., I. Noy-Meir, D. Renison, and M. Cabido. 2008. La ganadería extensiva , ¿ es compatible con la conservación de la biodiversidad y de los suelos? Ecología Austral 18:253-271.

Di Rienzo, J., F. Casanoves, B. Mónica, L. González, E. Tablada, and C. Robledo. 2008. InfoStat.

Eldridge, D. J., M. A. Bowker, F. T. Maestre, E. Roger, J. F. Reynolds, and W. G. Whitford. 2011. Impacts of shrub encroachment on ecosystem structure and functioning: towards a global synthesis. Ecology Letters 14:709-22. 
Eldridge, D. J., and S. Soliveres. 2015. Are shrubs really a sign of declining ecosystem function? Disentangling the myths and truths of woody encroachment in Australia. Australian Journal of Botany 62:594-608.

Gaitán, J. J., D. E. Bran, and F. Murray. 2007. Efecto de la severidad de quemado sobre la concentración de carbono orgánico en montículos e intermontículos en el monte austral. Ci Suelo 25:195-199.

Giorgetti, H. D., C. A. Busso, O. A. Montenegro, G. D. Rodríguez, and N. M. Kugler. 2006. Cattle Raising in Central, Semiarid Rangeland of Argentina. Rangeland Journal 28:32-36.

Godagnone, R. E., and D. E. Bran. 2009. Inventario integral de los recursos naturales de la provincia de Río Negro. INTA. Pp. 392.

González-Roglich, M., J. J. Swenson, D. Villarreal, E. G. Jobbágy, and R. B. Jackson. 2015. Woody Plant-Cover Dynamics in Argentine Savannas from the 1880s to 2000s: The Interplay of Encroachment and Agriculture Conversion at Varying Scales. Ecosystems 18:481-492.

Guevara, J. C., E. G. Grünwaldt, O. R. Estévez, A. J. Bisigato, L, J. Blanco, F. N. Biurrun, C. A. Ferrando, C. C. Chirino, E. Morici, B. Fernández, L. I. Allegretti, and C. B. Passera. 2009. Range and livestock production in the Monte Desert, Argentina. Journal of Arid Environments 73:228-237.

Kangas, A., and M. Maltamo. 2006. Forest inventory: Managing Forest Ecosystems, $1^{\circ}$. ed. Dordrecht, The Netherlands. Pp. 362.

Kröpfl, A. I., V. A. Deregibus, G. A. Cecchi. 2015. Un modelo de estados y transiciones para el Monte oriental rionegrino. Phyton 9457:390-396.

Kropfl, A. I., V. A. Deregibus, and G. A. Cecchi. 2007. Disturbios en una estepa arbustiva del Monte: cambios en la vegetación. Ecología Austral 17:257-268.

León, R. J. C., D. Bran, M. B. Collantes, J. M. Paruelo, and A. Soriano. 1998. Grandes unidades de vegetación de la Patagonia extraandina. Ecología Austral 8:125-144.

Maestre, F. T., S. Bautista, J. Cortina, and J. Bellot. 2001. Potential for using facilitation by grasses to establish shrubs on a semiarid degraded steppe. Ecological Applications 11:1641-1655.

Maestre, F. T., M. A. Bowker, M. D. Puche, M. Belén Hinojosa, I. Martínez, P. García-Palacios, A. P. Castillo, S. Soliveres, A. L. Luzuriaga, A. M. Sánchez, J. A. Carreira, A. Gallardo, and A. Escudero. 2009. Shrub encroachment can reverse desertification in semi-arid Mediterranean grasslands. Ecology Letters 12:930-41.

Mateucci, S., and A. Colma. 1982. Metodología para el estudio de la vegetacion. Monografía $\mathrm{N}^{\circ} 22$, serie Biología. Secretaría general de OEA. Washington D.C.

McNaughton, S. J. 1985. Ecology of a Grazing Ecosystem: The Serengeti. Ecological Monogrphs 55:259-294.

McNaughton, S. J. 1983. Serengeti Grassland Ecology: The Role of Composite Environmental Factors and Contingency in Community Organization. Ecological Monographs 53:291-320.

Morello, J., S. D. Mateucci, A. F. Rodríguez, and M. E. Silva. 2012. Ecoregiones y complejos ecosistémicos argentinos $1^{\circ}$ ed. Orientación Gráfica editora. Buenos Aires.

Mostacedo, B., and T. Fredericksen. 2000. Manual de métodos básicos de muestreo y análisis en ecología vegetal., $1^{\circ}$ ed. Santa Cruz de la Sierra.

Peter, G., F. A. Funk, and S. S. T. Robles. 2013. Responses of vegetation to different land-use histories involving grazing and fire in the North-east Patagonian Monte, Argentina. Rangeland Journal 35:273-283.

Rostagno, C. M., G. Defossé, and H. del Valle. 2006. Postfire Vegetation Dynamics in Three Rangelands of Northeastern Patagonia. Rangeland Ecology and Management 59:163-170.

Sala, O. E., F. S. III Chapin, J. J. Armesto, E. Berlow, J. Bloomfield, R. Dirzo, E. Huber-Sanwald, L. F. Huenneke, R. B. Jackson, A. Kinzig, R. Leemans, D. M. Lodge, H. A. Mooney, M. Oesterheld, L. Poff, M. T. Sykes, B. W. Walker, M. Walker, and D. H. Wall. 2000. Global Biodiversity Scenarios for the Year 2100. Science 287:1770-1774.

Shannon, C. E., and W. Wiener. 1949. The mathematical theory of communication. University of Illinois Press. Urbana, IL, EEUU. Pp. 144.

Soriano, A., and O. Sala. 1983. Ecological strategies in a Patagonian arid steppe. Vegetation 56:9-15.

Torres Robles, S. S., M. F. Arturi, C. Contreras, G. Peter, and J. M. Zeberio. 2015. Variaciones geográficas de la estructura y composición de la vegetación leñosa en el límite entre el Espinal y el Monte en el noreste de la Patagonia. Boletín de la Sociedad Argentina de Botánica 50:209-215.

Villagra, P. E., G. E. Defossé, H. F. del Valle, S. Tabeni, M. Rostagno, E. Cesca, and E. Abraham. 2009. Land use and disturbance effects on the dynamics of natural ecosystems of the Monte Desert: Implications for their management. Journal of Arid Environments 73:202-211.

Ward, D., K. Wiegand, and S. Getzin. 2013. Walter's two-layer hypothesis revisited: back to the roots. Oecologia 172: 617-630.

Zeberio, J. M. 2012. Avance de la frontera agropecuaria en el noreste patagónico y sus consecuencias en los procesos de desertificación y pérdida de biodiversidad. Pp. 216-221 in M. Dos Santos Afonso and T. S. Rosa (eds.). Ciencia y Tecnología Ambiental. Un Enfoque Integrador. Asociación Argentina para el progreso de la ciencia, Buenos Aires. 